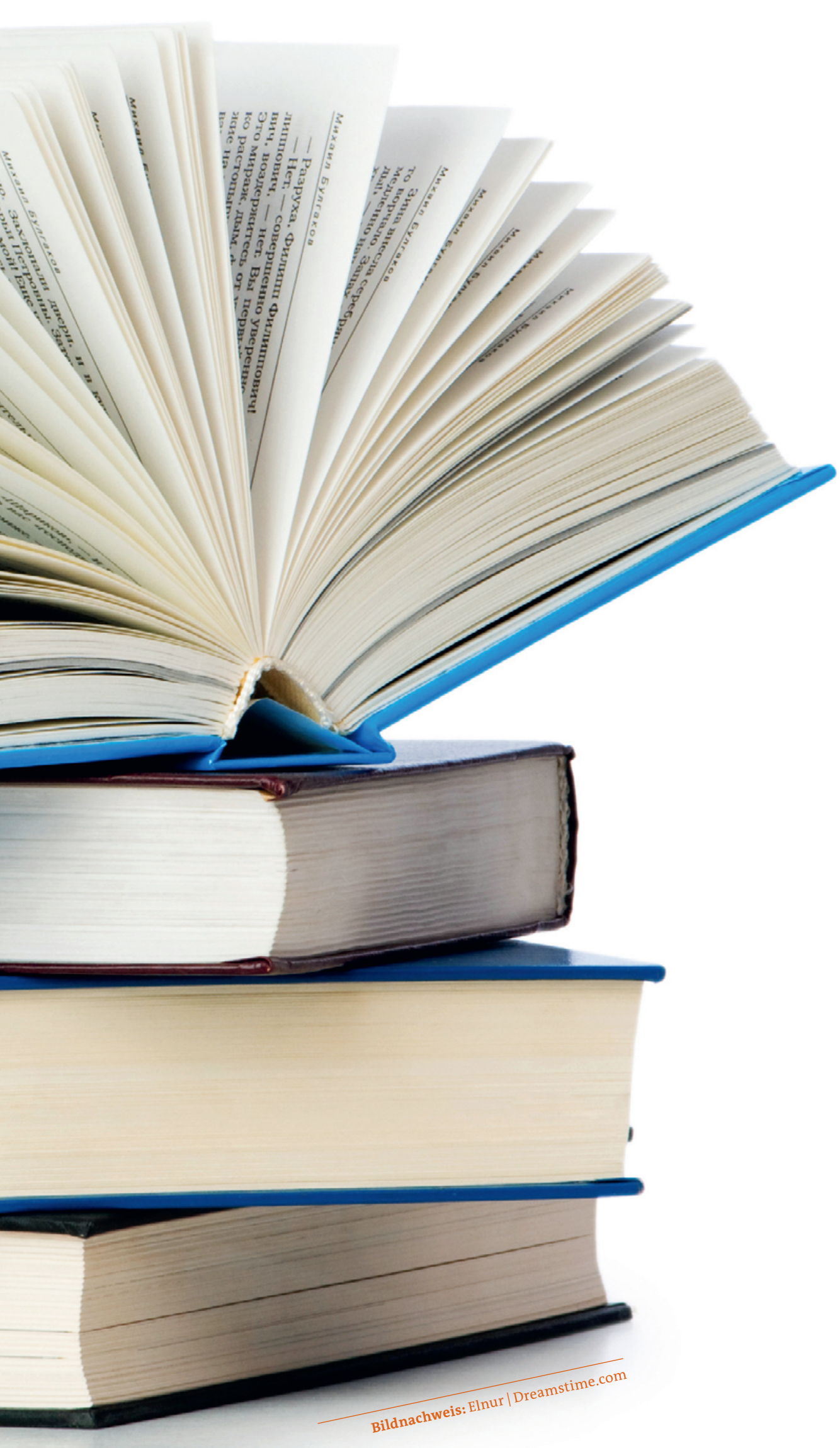

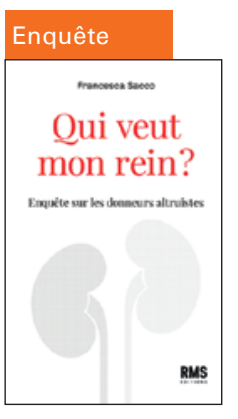

Qui veut mon rein?

Enquête sur les donneurs altruistes

Francesca Sacco

Chêne-Bourg: Médecine et Hygiène; 2021

Francesca Sacco, journaliste spécialisée dans la santé et la médecine, se penche sur la problématique des donneurs altruistes, soit celles et ceux qui sont prêts à donner un rein à n'importe quel receveur compatible - par solidarité, pour pallier le manque d'organes, ou "pour avoir une fois fait quelque chose de bien dans sa vie».

Cet ouvrage décrit la situation du don altruiste dans plusieurs pays, chiffres à l'appui. Rare en Suisse (un ou deux par an), il est interdit en Allemagne et en France: on sait les positions restrictives des Français liées à la non-patrimonialité du corps humain. Le don altruiste ne doit inclure aucun échange d'argent, point qui constitue une raison de réserve. Certains considèrent que ces donneurs, prêts à un geste «autosacrificiel», pourraient être des dérangés. Au long de l'ouvrage, les enjeux éthiques particuliers de ce don sont discutés, y compris avec un psychiatre lausannois.

Pour améliorer la qualité de la compatibilité entre donneur et receveur, les dons croisés de reins se sont beaucoup développés: au départ, entre deux paires de personnes avec des liens parentaux ou affectifs forts, mais où c'est le donneur de la paire A qui correspond le mieux au receveur de la paire $B$. Ce modèle a été élargi en mettant ensemble de multiples paires et des donneurs altruistes. C'est le cas en Suisse depuis 2019.

L'auteure a suivi Albert, candidat suisse au don altruiste depuis 2013. Les étapes de sa trajectoire sont décrites à travers plusieurs entretiens. Le livre se termine sur un échange avec lui au lendemain du prélèvement de son rein, en 2021: "Le chirurgien est venu me trouver. L'équipe de transplantation est enchantée, le rein était parfait, la greffe a bien pris. Nous avons tous gagné.» On découvre des récits de donneurs altruistes d'autres pays et ce qui les a motivés. Un chapitre présente même le «Portrait-robot du donneur altruiste».

Très informatif et agréable à lire, cet ouvrage est d'un grand intérêt pour qui suit les évolutions médicales et socio-éthiques.

Dr méd. Jean Martin membre de la rédaction 


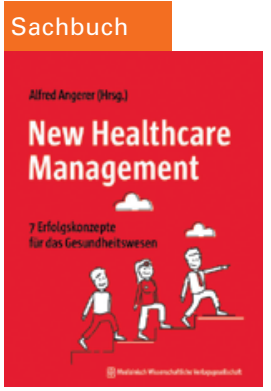

\section{New Healthcare Management} Alfred Angerer (Hrsg.)

Berlin: MWV; 2021
Selbstkritisch beginnt Alfred Angerer, Professor an der Zürcher Hochschule für Angewandte Wissenschaften, seine Einführung mit der Frage: «Noch ein Management-Buch?». Eigentlich gebe es davon ja schon genug. Doch der Herausgeber hat sich und seinen Co-Autorinnen und -Autoren zwei Ziele gesetzt, die dieses Buch besonders machen: Erstens soll es sich spezifisch an Führungskräfte im Gesundheitswesen richten und zweitens so eingängig sein, dass man es auch «am Strand» lesen kann. Vorgestellt werden sieben Erfolgskonzepte aus der Management-Literatur, die besonders zukunftsträchtig scheinen: Positive Führung, Value-based Healthcare, Design Thinking, Lean und Kaizen, Digital Health, Selbstorganisation und Agilität. Zusammen genommen würden diese Konzepte eine neue Form der Führung von Gesundheitsorganisationen, das titelgebende "New Healthcare Management», ermöglichen, doch sei jedes Konzept auch einzeln einsetzbar. Die Kapitel beginnen mit der Beschreibung frustrierender Organisationsabläufe oder veralteter Denkmuster, die sich in vielen Gesundheitseinrichtungen hartnäckig halten. Anschaulich und illustriert durch viele Abbildungen wird gezeigt, wieso und wie das jeweilige Konzept hier frischen Wind bringen kann.

Und während der Lektüre wird klar: Dieses Buch kann seine Versprechen einlösen. Es ist praxisnah, beleuchtet aktuelle Beispiele aus der Schweiz und Deutschland und richtet sich mit seinen Schritt-für-Schritt-Anleitungen direkt an Führungspersonen im Gesundheitswesen. Gleichzeitig ist der Stil durchgängig locker, immer leicht humorvoll und geprägt von den persönlichen Erfahrungen der Autorinnen und Autoren. So macht es Spass, «noch ein Management-Buch» zu lesen.

Rahel Gutmann, Junior-Redaktorin SÄZ

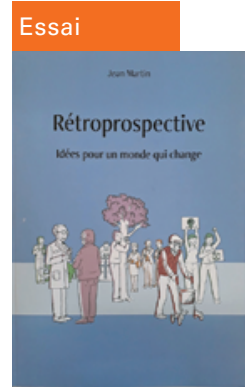

Rétroprospective

Idées pour un monde qui change

Jean Martin

Autoédition; 2021

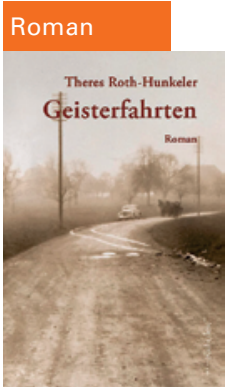

Geisterfahrten

Theres Roth-Hunkeler

Luzern: edition

bücherlese; 2021

Ce recueil, compilation de textes récents publiés, est bien rétro-prospectif, comprenant tout autant une traversée qu'un programme. Une traversée: celle de divers mouvements sociétaux touchant entre autres à la liberté d'expression, à la biodiversité et au climat, aux enjeux de la procréation médicalement assistée, aux inégalités sociales, à l'accompagnement en fin de vie, ainsi qu'à l'influence des lobbys et des conflits d'intérêt, dont notre collègue Jean Martin a tenu très régulièrement la chronique, avec une curiosité vive et engagée. Ces pages empreintes de tolérance rappellent combien ces mutations de la société interpellent de près la médecine et son éthique, ceci parfois même âprement. Elles ne cherchent cependant pas à masquer la contrariété et l'inquiétude que peuvent induire le climato-scepticisme, le manque de solidarité ou la dévalorisation du statut de la vérité.

L'ouvrage dessine également un programme. Parmi les pistes, il est suggéré de tenir compte des interdépendances et d'en prendre soin, d'analyser les enseignements de l'histoire et aussi - surtout? - d'admettre comme inéluctable un changement général du modèle de société. Il contient également un credo, voire un appel: il n'est plus tenable aujourd'hui de se retrancher derrière une neutralité dogmatique. "Indignez-vous!» a été l'injonction très médiatisée de l'écrivain Stéphane Hessel. "J'aime ce monde, il faut le changer", répond ici Jean Martin avec sa plume engagée et courtoise.

Prof. Dre méd. Anne-Françoise Allaz, membre de la rédaction

Cette publication peut être obtenue auprès de Jean Martin, au prix de CHF 25 en s'adressant à jeanmartin280[at]gmail.com

\section{Teilen Sie Ihre literarischen Entdeckungen mit uns!}

Sie haben ein interessantes Buch gelesen und möchten es einem weiteren Leserkreis vorstellen?

Schicken Sie uns Ihre Buchbesprechung (max. 1200 Zeichen inkl. Leerzeichen) an: redaktion.saez[at]emh.ch
Sich den Durchblick in einer Familiengeschichte zu verschaffen ist alles andere als einfach. Diese Erfahrung macht jede Ärztin, jeder Arzt bei der Familienanamnese. In jeder Familie gibt es dunkle Ecken und herumirrende Geister. Von ihnen wird Lisa bedrängt. Sie ist eine frisch pensionierte Hotelangestellte und möchte nun endlich erfahren, wie ihr Halbbruder und dessen Mutter ums Leben kamen. An einem regnerischen Tag im November 1938 kam es zur Kollision zwischen dem Auto eines Tierarztes und einer jungen Mutter mitsamt ihrem kranken Söhnchen im Kinderwagen - sie wurden überfahren und kamen zu Tode. Dieses Ereignis ist protokolliert und einsehbar in den Gerichtsakten des Kantons Luzern. Es bildet den einen Teil des Romans Geisterfahrten. Die Geschichte von Lisa und ihren Erkundigungen hingegen ist erfunden.

Erfunden von Theres Roth-Hunkeler, die mit diesem Titel ihren sechsten Roman vorlegt. Hier zeigt die Autorin präzis und anschaulich, was alles unterschwellig vorhanden ist an Erwartungen, Verflechtungen und Abstossungen im System «Familie». Lisa hofft auf Ernst, ihren zweiten Halbbruder, der einiges älter ist als sie und etwas über die Vorkommnisse von damals erzählen könnte. Sie arrangiert gemeinsame Ferien mit ihm im Tessin. Damit aber gerät Lisa auf eine Achterbahn von unvorhergesehenen Ereignissen, unauslöschlichen Erinnerungen und verwirrenden $\mathrm{Ge}$ fühlen. Und mit ihr unweigerlich auch die Leserinnen und Leser dieses vielschichtigen Romans.

Dr. med. Felix Schürch, Praxis Schürch, Zürich

\section{Partagez vos découvertes littéraires!}

Vous avez lu un ouvrage intéressant et souhaitez en faire profiter d'autres lecteurs? Envoyez-nous votre critique littéraire (max. 1200 signes, espaces compris) à: redaction.bms[at]emh.ch 